\title{
A New Sex-Specific Genetic Map of the Human Pseudoautosomal Regions (PAR1 and PAR2)
}

\author{
Antònia Flaquer ${ }^{a, c}$ Christine Fischer ${ }^{b}$ Thomas F. Wienker ${ }^{a}$ \\ ${ }^{a}$ Institute for Medical Biometry, Informatics and Epidemiology, University of Bonn, Bonn, ${ }^{b}$ Institute of \\ Human Genetics, University of Heidelberg, Heidelberg, and 'Institute of Medical Biometry and Epidemiology, \\ Philipps University Marburg, Marburg, Germany
}

\section{Key Words}

Pseudoautosomal regions $\cdot$ Genetic maps · Multipoint

linkage analysis $\cdot$ Recombination rate $\cdot$ Crossovers

\begin{abstract}
Objectives: Accurate sex-specific genetic maps are essential for gene mapping projects when using multipoint methods, especially for the pseudoautosomal regions (PARs). This paper describes a new sex-specific genetic map for the human PARs. Methods: We used multipoint linkage analysis, threegeneration pedigrees and to date, the largest set of genetic markers to estimate recombination events in the PARs. The new map combines genotype data from $28 \mathrm{CEPH}$ pedigrees and 29 genetic markers. All genetic markers have been physically mapped, thus providing the opportunity to compare genetic to physical distance through all the intervals. $\boldsymbol{R e}$ sults: Recombination activity in PAR1 is on average 7-fold higher in male than in female meiosis, resulting in a much larger genetic map for males. This new map shows how genetically different in size is the $X$ chromosome between males and females. Conclusions: Genetic distances vary extremely between males and females in the PARs. These differences should be considered in genetic studies using accurate sex-specific maps instead of sex-averaged maps. The new map provides a significantly different length compared
\end{abstract}

to some previously published maps, since a much higher number of genetic markers is used. Therefore, we conclude that our map is the most accurate resource for obtaining genetic map information for the PARs.

Copyright $\odot 2009$ S. Karger AG, Basel

\section{Introduction}

Human sex chromosomes, $\mathrm{X}$ and $\mathrm{Y}$, are morphologically and genetically different. During male meiosis pairing and crossover take place in two small regions on both the short and the long arm adjacent to the telomeres, the pseudoautosomal regions (PAR1 and PAR2) [1, 2]. Their physical lengths are $\sim 2.7 \mathrm{Mb}$ for PAR1 and $\sim 0.33 \mathrm{Mb}$ for PAR2. The pseudoautosomal boundaries (PAB1, PAB2) separate highly recombinant regions from non-recombinant regions on the $\mathrm{Y}$ and a moderately recombining region on the $\mathrm{X}$ chromosome. For more detailed information about the human PARs we refer to the recent review by Flaquer et al. [3].

To date, 24 genes have been reported in the PAR1 and 5 genes in the PAR2 [4]. The only known disease gene within the PARs is the Short Stature Homeobox (SHOX) gene, functional loss of which is causally related to various short stature conditions and disturbed bone develop-

\section{KARGER}

Fax +4161306 1234 E-Mail karger@karger.ch www.karger.com
(C) 2009 S. Karger AG, Base

0001-5652/09/0683-0192\$26.00/0

Accessible online at:

www.karger.com/hhe
Antònia Flaquer

Institute of Medical Biometry and Epidemiology

Philipps University Marburg

Bunsenstrasse 3, DE-35032 Marburg (Germany)

Tel. +496421 286 6294, Fax +496421 286 8921, E-Mail flaquer@staff.uni-marburg.de 
Fig. 1. Published genetic maps for PAR1: Relations among the sex-specific genetic maps proposed so far for PAR1. Different techniques were used for the estimation of recombination rates, three-generation families (FAM) and sperm cell typing (SPR). Samples used: Henke (38 CEPH pedigrees, 11 markers) $[12,13]$, Rouyer (8 CEPH pedigrees, 5 markers) [14], Lien (1912 sperm cells from 4 donors, 9 markers) [15], Schmitt (900 sperm cells from 2 donors, 4 markers) [16]. Rutgers (42 CEPH pedigrees, 7 markers) [17]. Note that the graphs use a different scale for males and females. Obviously, the maps based on SPR techniques are only available for males.

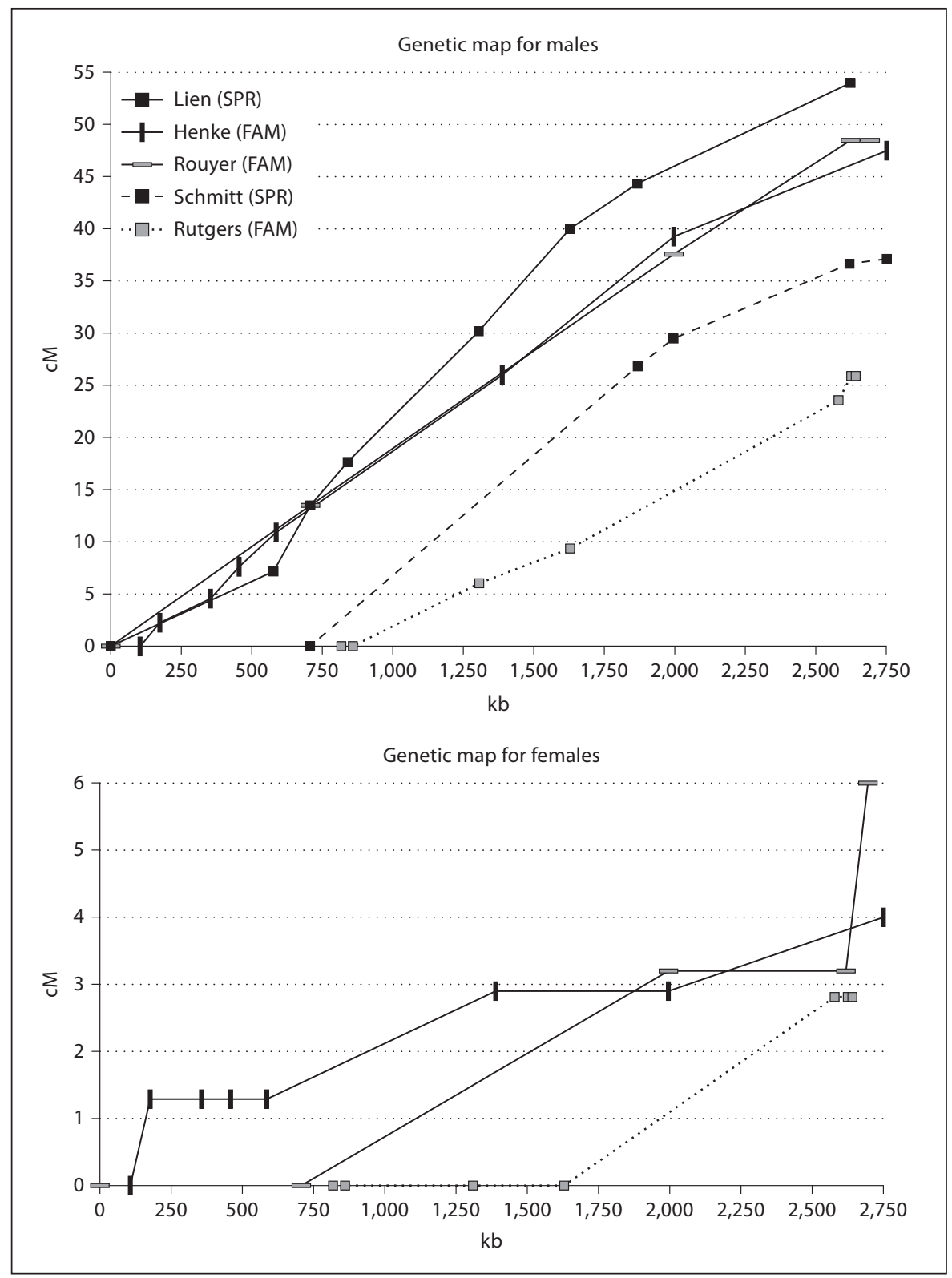

ment, recently reviewed by Blaschke et al. [5]. However, the PARs are largely ignored in systematic genome-wide linkage and association analysis.

Accurate and comprehensive genetic maps are crucial for the success of several types of genetic studies such as linkage analysis, positional cloning projects and even for some aspects of genome-wide association analysis [6-8]. The order and physical location of the majority of genetic markers can be determined from the assembled sequence of the human genome. However, unless a given group of markers are all present on a single genetic map, specification of recombination-based genetic map distances for any large set of markers remains difficult. In theory, physical map distances can be used to interpolate and estimate genetic maps distances. However, the intense variability in the distribution of recombination rates in the genome needs a particular effort to identify properly region-specific estimates of recombination intensity $\mathrm{cM} / \mathrm{kb}$, resulting in large-scale interpolation generally unfeasible. Accurate estimates of map distances can only be obtained by multipoint linkage analysis using genotype data. 
Genetic Maps for the PARs

A few genetic maps have been proposed so far for the PARs. They have been constructed using the techniques of three-generation families, unrelated individuals or sperm cells to estimate recombination rates [9-11]. Figure 1 illustrates the relations among the published genetic maps for males and females in PAR1. The genetic maps for males proposed by Lien, Henke and Rouyer seem to be well in agreement for the first $750 \mathrm{~kb}$. The discordance shown by Schmitt's and Rutgers' map could be because of the most telomeric marker still being at a distance of approximately $750 \mathrm{~kb}$ from the telomere. After $750 \mathrm{~kb}$, the maps display different tendencies, only Rouyer's map and Henke's map display a similar trend.

The differences among these published maps could be due to a systematic map estimation bias, data errors, or an insufficient number of markers or meioses considered for the estimation of the recombination rate. These differences are less significant when comparing the genetic maps proposed for females. Based on these maps the estimated genetic length of PAR1 varies between 26 and 54 $\mathrm{cM}$ in males and between 2.8 and $6 \mathrm{cM}$ in females. There is a very small number of genetic maps proposed for PAR2, and all of them used a poor number of genetic markers, recently reviewed by Flaquer et al. [3].

It is true that the international HapMap project used a much larger number of genetic markers (1400 SNPs in PAR1 and 140 SNPs in PAR2) to estimate a genetic map [18]. This map was based on the technique of unrelated individuals providing only sex-averaged distances. To be comparable with the other maps, we computed sex-specific map distances using a male/female map ratio of 10:1 (data not shown). HapMap estimates were lower in respect to the other maps, this could be because using the technique of unrelated individuals is very difficult to estimate accurate sex-specific recombination rates. With this technique a coalescent model of a finite neutrally evolving population with constant population size is assumed which cannot be easily confirmed with empirical data, and only sex-averaged recombination rates can be estimated. We do not present the map here because recently the new genetic map from HapMap phase 3 has become available where the sex chromosomes, and hence the PARs, have been excluded.

Multipoint linkage analysis is the standard tool in the search for genetic variants that predispose to Mendelian and complex genetic diseases, since this method is generally more powerful than single point methods. However, a genetic map is required for multipoint analysis. Misspecification of the genetic map has the potential to se- verely compromise the estimation and testing procedures used in multipoint linkage analysis. Specifically, loss of power to detect linkage or an increase in the false positive rate, and bias in the disease locus position estimate are possible $[19,20]$. Genetic map misspecification could occur from over-simplified models of the biological process of recombination in a genetic region. Notably, variation in recombination rates between males and females indicates that sex-averaged maps omit significant information about sex-specific genetic distances. Due to the high intensity of recombination events in the PARs, a large number of genetic markers should be considered to estimate genetic maps for these regions.

To help to address these issues, we have created a sexspecific genetic map using the technique of multipoint linkage analysis and three-generation $\mathrm{CEPH}$ pedigrees (Centre d'Etude du Polymorphisme Humain) to estimate recombination rates, including the largest set, to the best of our knowledge, of genetic markers in PAR1 and PAR2.

\section{Materials and Methods}

\section{Genotypes and CEPH Pedigrees}

Genotypes for the PARs were obtained from the CEPH genotype database and from the Department of Genetics of the Rutgers University [17]. We selected CEPH pedigrees where pseudoautosomal genotypes were available for three-generation pedigrees. Altogether, 23 and 6 genetic markers were available for PAR1 and PAR2 respectively for a total of $29 \mathrm{CEPH}$ pedigrees. To identify genotypes that lead to non-Mendelian transmission and likely to be erroneous, as well as to search for problematic pedigrees, the PEDCHECK program was used [21]. However, all these genotype data have been previously cleaned, either by the groups who determined the genotypes or by other groups who have used the data. Thus, no Mendelian inconsistencies were found and no problematic pedigrees were detected. Anyway, it is well known that some errors do not show up as Mendelian inconsistencies. Suppose for example that two parents with genotypes $1 / 2$ and $2 / 3$ in a locus give birth to a child with genotype $1 / 2$, but for some error the child appears in the database with genotype $1 / 3$, the child is still consistent with the parents and this error will not be detected as a Mendelian inconsistency. To check for this type of errors a procedure based on recombination events was used. It has been argued whether multiple crossover events could occur within the small PARs. Two studies reported a double crossover event in male meiosis and to date no double crossover event has been reported in female meiosis $[16,22]$. In this study we found one double recombination event in male meiosis and seven in female meiosis. The seven double recombination events in female meiosis were regarded as a warning to potential genotype errors. We took a closer look at those events and they all were from seven different individuals within the same family (FAM-66). There was no clear-cut and obvious solution to this problem. The perfect solution would be to regeno- 


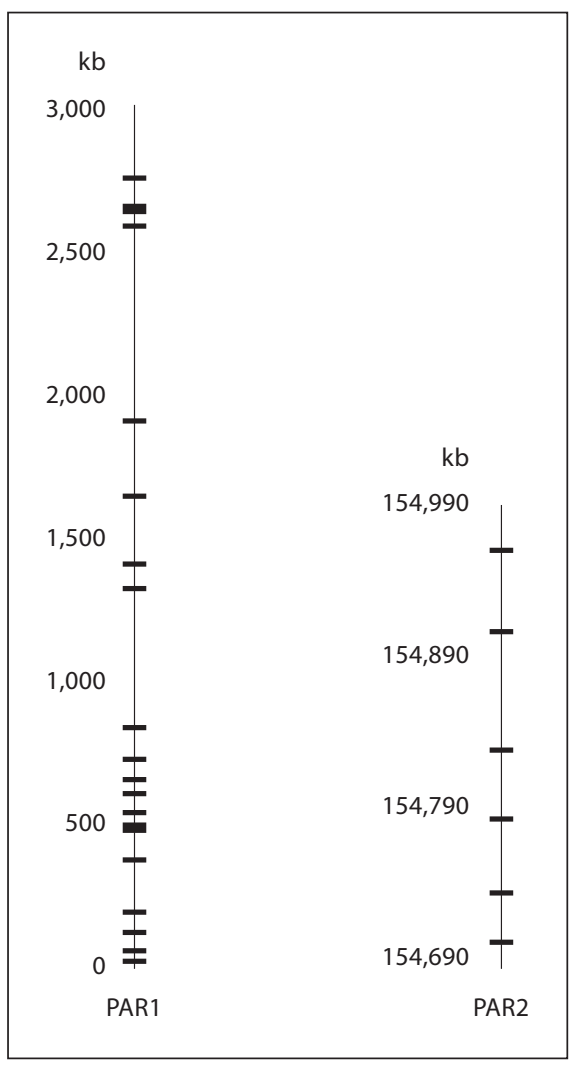

Fig. 2. Position and density of the genetic markers: illustrates the position and density of the genetic markers used to construct the genetic map in PAR1 and PAR2. Note that the graphs for PAR1 and for PAR2 do not use the same scale.

Table 1. Characteristics of the $28 \mathrm{CEPH}$ pedigrees used to estimate the sex-specific genetic maps of PAR1 and PAR2

\begin{tabular}{ll}
\hline 28 CEPH families & $\mathrm{n}$ \\
\hline Individuals & 413 \\
Founders & 112 \\
Non-founders & 301 \\
Females & 202 \\
Males & 211 \\
Pedigree size & $13-21$ \\
Generations & 3 \\
\hline
\end{tabular}

type this family. For the time being we decided to exclude this family from the analysis.

At the end, $28 \mathrm{CEPH}$ pedigrees were considered in the analysis including families: 13291-13294, 1331-1334, 1340, 1341, 1344, $1346,1347,1349,1350,1362,1375,1377,1413,1416,1418,1420$, $1421,1423,1424,12,104$ and 884 . The characteristics of these pedigrees are summarized in table 1.

A Genetic Map for the Pseudoautosomal Regions

\section{Genetic Markers}

The characteristics of the 28 genetic markers (22 in PAR1, 6 in PAR2) used to construct the genetic maps reported in this study are summarized in table 2 . These include the protein coding gene colony stimulating factor 2 receptor alpha, CSF2RA, as well as 19 other microsatellite (STR) markers and 8 SNPs. Locus DXYS225 located in PAR2 is closely linked to the protein coding gene synaptobrevin-like 1, SYBL1, and locus DXYS227 is very near to the protein coding gene interleukin 9 receptor, IL9R. At the end of PAR1 and thus effectively at the PAB1, sex as a phenotypic marker was added to represent the SRY (sex determiner) gene. For this marker females were denoted as homozygous with alleles $1 / 1$ and males as heterozygous $1 / 2$. Heterozygosity percentages have been calculated from the genotypes of unrelated individuals from the $28 \mathrm{CEPH}$ pedigrees $(\mathrm{n}=112)$. Probes from PAR1: TSC0240426, TSC0240423 and TSC0551442, and probes from PAR2: TSC0268423 and TSC0897419 were obtained from the Department of Genetics of the Rutgers University. The rest of the probes are publicly available from the CEPH database.

A concerned effort was made to ensure the uniqueness of the markers in both sets. A comparison of marker name alias was used to identify markers that were represented in the data set more than once. Whenever possible, multiple lines of evidence, including comparison of physical positions, were sought to confirm and identify redundancy. These redundancies included only two markers that were identified by alias names. For these duplicated markers the one with a higher call rate was included in the analysis. One marker, DXYS20, matched more than two positions on contig(s) by ePCR. The option Build from CRI-MAP was used to identify the most likely location [23]. With this option the marker is placed in each possible interval between two flanking markers in the map. The resulting orders are then tested for compatibility with the database. Each order not excluded is subjected to a full maximum likelihood estimation. The order having the highest $\log 10$ likelihood is found, any order whose log10 likelihood is less than this one by more than a specified tolerance is eliminated. Using this method DXYS20 was placed between GATA151F04 and DXYS78 supporting the location given by Henke et al. [12]. In the end, a set of 23 and 6 loci that, to the best of our knowledge, represent non-redundant loci within PAR1 and PAR2, respectively, were used to estimate the sex-specific genetic map in the PARs.

The physical position of the genetic markers employed in each interval are represented in figure 2. To improve the accuracy of the maps, the genomic sequence position for the 29 markers were readily identified from the Ensembl Genome browser, the $\mathrm{Na}$ tional Center for Biotechnology Information (NCBI) and/or from the dbSNP database.

\section{Statistical Analysis}

Genetic markers at the PARs follow the same inheritance pattern as autosomal markers, becoming progressively more sexlinked (i.e. recombination events are becoming more rare between $\mathrm{X}$ and $\mathrm{Y}$ ) as they approach to the PAB. Therefore genetic distances can be estimated with standard techniques and computer programs for linkage analysis. Recombination rates are to be estimated for both sexes separately, since a marked difference in recombination frequency exists between males and females. To establish the genetic maps the software CRI-MAP v2.4 which allows rapid largely automated construction of multipoint linkage 
Table 2. Genetic markers included in the analysis to estimate the sex-specific genetic map for PAR1 and PAR2

\begin{tabular}{|c|c|c|c|c|c|c|}
\hline PAR & Locus & Probe & Enzyme & $\begin{array}{l}\text { No. of } \\
\text { alleles }\end{array}$ & $\begin{array}{l}\text { Hetero- } \\
\text { zygosity, \% }\end{array}$ & $\begin{array}{l}\text { No. of informative } \\
\text { meioses (female/male) }\end{array}$ \\
\hline 1 & DXYS14 & CEB12 & Pvull & 4 & 79.8 & $364(175 / 189)$ \\
\hline 1 & GATA151F04 & GATA151F04B & pcr & 10 & 63.6 & $105(59 / 46)$ \\
\hline 1 & DXYS20 & $3 \cos -\mathrm{PP}$ & Taql & 8 & 91.4 & $333(182 / 151)$ \\
\hline 1 & DXYS78 & cMS600 & Taql & 4 & 98.4 & $395(199 / 196)$ \\
\hline 1 & DXYS60 & U7A & EcoRI & 2 & 50.5 & $19969 / 130)$ \\
\hline 1 & DXYS87 & P99 & Taql & 3 & 60.8 & $267(148 / 119)$ \\
\hline 1 & DXYS161 & B6-Pol & Taql & 4 & 66.7 & $275(130 / 145)$ \\
\hline 1 & D0S17712 & UT708 & $\operatorname{pcr}(n)$ & 4 & 72.1 & $84(32 / 52)$ \\
\hline 1 & DXYS28 & pDP411a & Taql & 4 & 50.8 & $213(106 / 107)$ \\
\hline 1 & DXYS162 & AK1 & Taql & 8 & 91.8 & $376(180 / 196)$ \\
\hline 1 & GATA42G01 & GATA42G01 & pcr & 5 & 67.5 & $132(51 / 81)$ \\
\hline 1 & DXYS15 & $113 \mathrm{D}$ & Taql & 7 & 73.0 & $181(85 / 96)$ \\
\hline 1 & DXYS233 & AFMa284xc9 & $(\mathrm{AC}) \mathrm{n}$ & 11 & 85.2 & $274(150 / 124)$ \\
\hline 1 & DXS9900 & GGAT3F08 & PCR & 5 & 69.2 & $114(54 / 60)$ \\
\hline 1 & CSF2RA & CSF2RA & Taql & 8 & 83.5 & $361(180 / 181)$ \\
\hline 1 & DXYS234 & AFM273xb9 & $(\mathrm{AC}) \mathrm{n}$ & 8 & 67.5 & $129(50 / 79)$ \\
\hline 1 & DXYS89 & MS639 & Hinfl & 4 & 50.1 & $203(100 / 103)$ \\
\hline 1 & rs2267 & WIAF-2278 & SNP & 2 & 30.2 & $64(38 / 26)$ \\
\hline 1 & rs924904 & TSC0240426 & SNP & 2 & 41.9 & $178(64 / 114)$ \\
\hline 1 & rs310136 & TSC0240423 & SNP & 2 & 14.7 & $53(22 / 31)$ \\
\hline 1 & rs311071 & TSC0551442 & SNP & 2 & 41.0 & $161(71 / 90)$ \\
\hline 1 & rs3671 & WIAF-2434 & SNP & 2 & 17.2 & $47(34 / 13)$ \\
\hline 2 & rs802488 & TSC0440751 & SNP & 2 & 35.0 & $132(45 / 87)$ \\
\hline 2 & DXYS154 & SDF1 & pcr & 8 & 76.3 & $118(59 / 59)$ \\
\hline 2 & DXYS225 & LH1 & $\mathrm{pcr}$ & 2 & 51.7 & $28(14 / 14)$ \\
\hline 2 & DXYS227 & $\mathrm{LH} 2$ & pcr & 4 & 69.0 & $22(8 / 14)$ \\
\hline 2 & rs963311 & TSC0268423 & SNP & 2 & 49.0 & $258(115 / 143)$ \\
\hline 2 & rs188305 & TSC0897419 & SNP & 2 & 48.0 & $245(110 / 135)$ \\
\hline
\end{tabular}

maps was used [23]. CRI-MAP deduces missing genotypes where possible, and computes a likelihood based only on analysis of the known or deduced genotypes. Once the recombination fraction was estimated for each interval, it was converted into cM applying the identity function. The identity function (1\% recombination rate corresponds to $1 \mathrm{cM}$ ) has been suggested to be the most suitable mapping function for the PARs [3]. In order to evaluate whether our map is statistically different from previously suggested maps we performed statistical analysis of differences between recombination rates, including pairwise comparisons by a two-tailed Z-test.

\section{Results}

The multipoint genetic map for PAR1 was estimated using a total of 23 genetic markers (including the sex marker) and a total of 6 genetic markers for PAR2. For each region $28 \mathrm{CEPH}$ pedigrees were analyzed to estimate recombination rates separately for males and females. In determining the haplotypes of 245 offspring, 112 recombination events in male meioses were observed and 12 recombination events in female meioses. A double recombination event in male meiosis took place in family 13294 for individual 5. This double event was analyzed cautiously for a potential genotype error. We decided to accept it after finding out that this event could be a replication of the double event found by Rappold et al. in the same individual [22]. It was considered a replication after determining that the genotypes came from different labs and were genotyped by different methods. The resulting genetic maps, for PAR1 and PAR2, are illustrated in figure 3. Black dots represent the estimated genetic map for males, and gray dots for females. A high rate of recombination in female meiosis is seen only at the $\mathrm{Xp}$ telomere within the first $100 \mathrm{~kb}$. Within this telomeric region no difference in male and female recombination rates are observed, resulting in similar genetic distances for both sexes. After $100 \mathrm{~kb}$, genetic distances are becoming increas- 


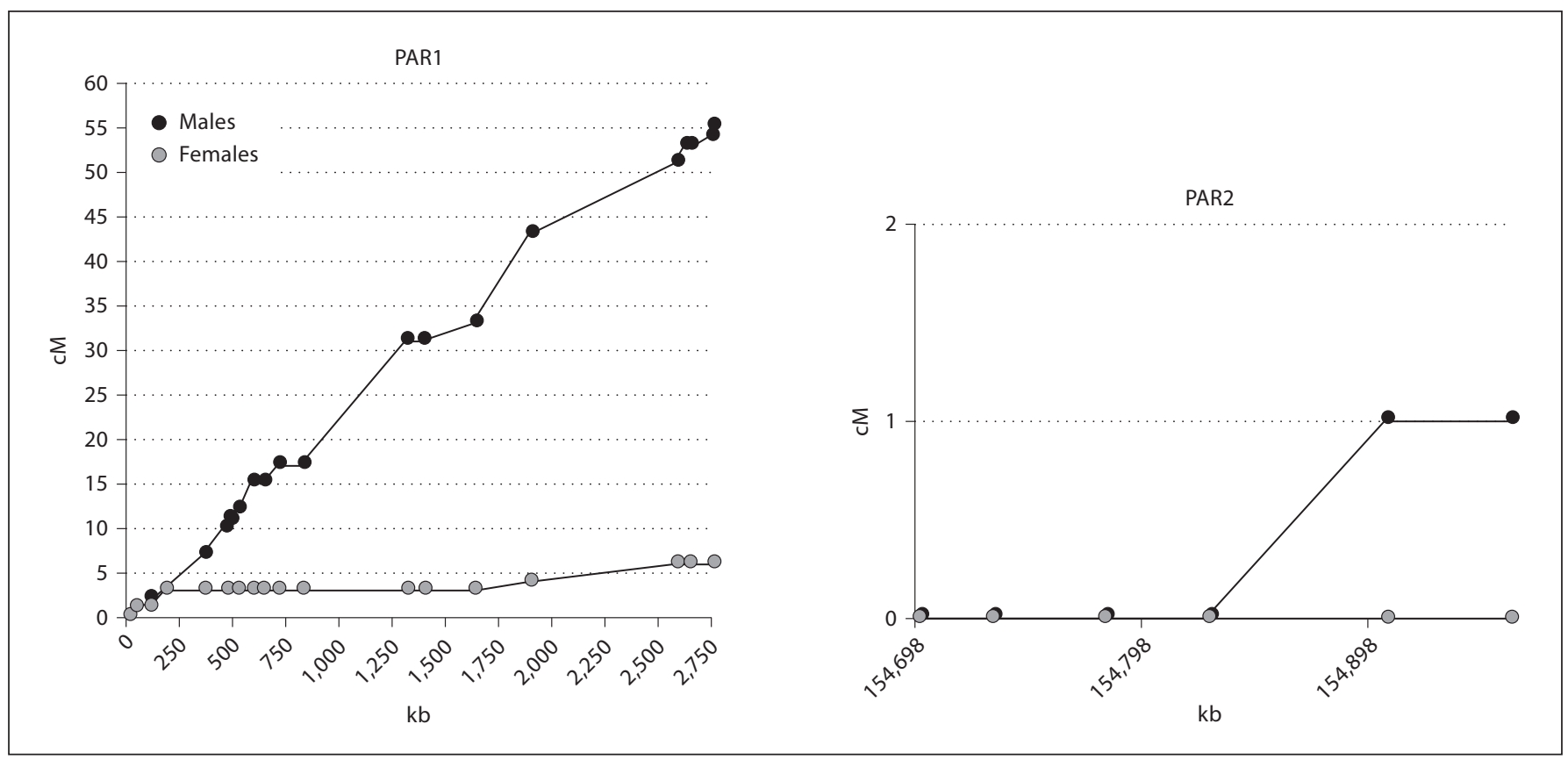

Fig. 3. Genetic map for PAR1 and PAR2: illustrates the positions of the estimated genetic maps (cM) in relation of the physical map (bp) for PAR1 and PAR2. Black points represent the genetic positions for males and gray points for females.

ingly different between males and females as they approach the PAB1. The estimated length of PAR1 is $55 \mathrm{cM}$ for males and $6 \mathrm{cM}$ for females. On the other hand, PAR2 does not seem to be significantly different in terms of recombination events between males and females, resulting in similar genetic maps. We detected one recombination event in male meioses and none in female meioses. These numbers are in the range suggested by Freije et al. [2].

The resulting genetic length for PAR1 was compared with the genetic lengths provided by each of the previously published maps. We also compared the genetic length in three subintervals of PAR1, close to the telomere $(\sim 0.9-580 \mathrm{~kb})$, in the middle of PAR1 ( 580-1628 kb) and close to the PAB1 ( $\sim 1628-2750 \mathrm{~kb})$. Pairwise Z-tests (two-tailed) were used to statistically establish differences in recombination rates. $p$ values from the Z-tests after Bonferroni correction are illustrated in table 3. When comparing the entire genetic length of PAR1 in males, large significant differences were detected with respect to the genetic lengths suggested by Schmitt and Rutgers $\left(p_{\text {Schmitt }}=2.6 \times 10^{-6}, p_{\text {Rutgers }}=4.9 \times 10^{-11}\right)$. The significant difference shown in the genetic length suggested by Henke is at border line $\left(p_{\text {Henke }}=0.04686\right)$. The genetic lengths proposed by Rouyer and by Lien did not show statistical differences with respect to the new genetic length although Lien's map displayed a significant shorter genetic length close to the telomere and close to the PAB1 $\left(p_{\text {Lien }}=3.6 \times 10^{-6}, p_{\text {Lien }}=0.00460\right.$, respectively $)$. When comparing the genetic length in females the Rutgers' map was the only one to show a slightly shorter length ( $\left.p_{\text {Rutgers }}=0.04942\right)$.

The map obtained for PAR2 was not statistically compared with the other maps since only one recombination event was detected in male meiosis. This is in the same range as in the already published maps for PAR2 [3].

The relation between the genetic and physical map of the male and female $\mathrm{X}$ chromosomes inferred from this study is represented in figure 4 . The X-specific data is from the study, 'A second-generation combined linkagephysical map of the human genome' [17].

\section{Discussion}

The pseudoautosomal regions of the human sex chromosomes reflect a higher recombination rate per physical length unit compared to the rest of the X chromosome. Using a total of 29 different genetic markers (23 in PAR1 and 6 in PAR2), we generated high resolution maps for the two PARs of the sex chromosomes. While the entire 
Fig. 4. Genetic and physical map for the human X chromosome: represents the relation between the genetic and physical map of the human X chromosome. The Xspecific data is from the Rutgers map [17]. PAR1 and PAR2 positions are based on the estimated genetic map of this work.

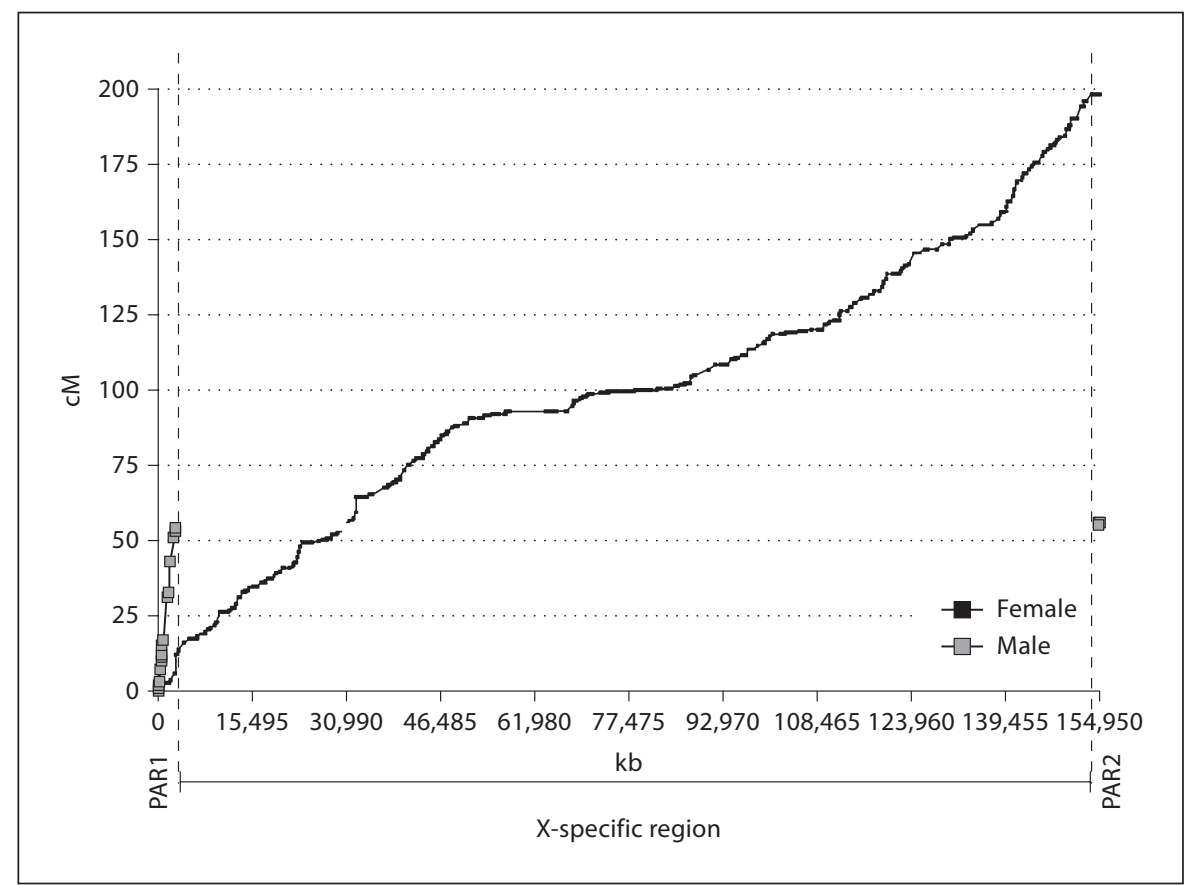

Table 3. Comparison of recombination fractions between our new map and each of the published maps for PAR1

\begin{tabular}{|c|c|c|c|c|c|c|c|c|c|c|c|}
\hline \multirow[t]{2}{*}{ Length $(\sim \mathrm{kb})$} & \multirow[t]{2}{*}{$\theta_{\text {new }}$} & \multicolumn{2}{|l|}{ Lien } & \multicolumn{2}{|c|}{ Rouyer } & \multicolumn{2}{|c|}{ Schmitt } & \multicolumn{2}{|c|}{ Henke } & \multicolumn{2}{|c|}{ Rutgers } \\
\hline & & $\theta$ & $\mathrm{p}$ value & $\theta$ & $\mathrm{p}$ value & $\theta$ & $\mathrm{p}$ value & $\theta$ & $\mathrm{p}$ value & $\theta$ & $\mathrm{p}$ value \\
\hline \multicolumn{12}{|l|}{ Males } \\
\hline $0.9-2,750$ (entire PAR1) & 0.55 & 0.54 & 0.39621 & 0.49 & 0.15836 & 0.37 & $2.6 \times 10^{-6}$ & 0.48 & 0.04686 & 0.26 & $4.9 \times 10^{-11}$ \\
\hline $0.9-580$ & 0.17 & 0.07 & $3.6 \times 10^{-6}$ & 0.11 & 0.30521 & n.c. & - & 0.11 & 0.08592 & n.c. & - \\
\hline $580-1,628$ & 0.16 & 0.33 & 1.00000 & 0.22 & 1.00000 & 0.21 & 1.00000 & 0.21 & 1.00000 & 0.09 & 0.04332 \\
\hline $1,628-2,750$ & 0.22 & 0.14 & 0.00460 & 0.16 & 0.37488 & 0.16 & 0.05515 & 0.16 & 0.18004 & 0.17 & 0.19864 \\
\hline \multicolumn{12}{|l|}{ Females } \\
\hline $0.9-2,750$ (entire PAR1) & 0.06 & n.a. & - & 0.06 & 0.50000 & n.a. & - & 0.04 & 0.17308 & 0.03 & 0.04942 \\
\hline $0.9-585$ & 0.03 & n.a. & - & 0.00 & 0.15139 & n.a. & - & 0.01 & 0.31923 & n.c. & - \\
\hline $585-1,628$ & 0.00 & n.a. & - & 0.02 & 1.00000 & n.a. & - & 0.02 & 1.00000 & 0.00 & 1.00000 \\
\hline $1,628-2,750$ & 0.03 & n.a. & - & 0.04 & 1.00000 & n.a. & - & 0.01 & 0.22349 & 0.03 & 1.00000 \\
\hline
\end{tabular}

$\theta_{\text {new }}$ refers to the recombination fraction estimated in this work. n.c. $=$ Not covered (genetic markers in this interval were not used). n.a. = Not available (map estimates based on sperm cells are only available for males).

$\mathrm{p}$ values from $\mathrm{Z}$-test (two-tailed) after Bonferroni correction for multiple testing. Hypothesis test for differences between two proportions $\left(\mathrm{H}_{0}: \theta_{\text {new }}=\theta\right.$ vs. $\left.\mathrm{H}_{1}: \theta_{\text {new }} \neq \theta\right)$.

PAR1 may be seen as a recombination hot spot in male meiosis, this is not the case in female meiosis. We obtained on average a recombination frequency 7 -fold higher in male meiosis compared to female meiosis. The climax arises close to the PAB1 where the recombination rate is 10 -fold higher in males than females. These large differences in recombination rates are translated into different genetic map lengths for males and females. Similar genetic lengths are observed only at the Xp telomere within the first $100 \mathrm{~kb}$. On the other hand, PAR2 does not seem to be significantly different in terms of genetic lengths between males and females. We detected only one 
recombination event in male meiosis. So far, all studies in PAR2 agree that no crossover events occur in females. However, this should not lead to the conclusion that PAR2 is an 'iced' recombination spot in female meiosis. Indeed, studies with a higher number of meioses are required to see the real behavior of PAR2.

It should be mentioned that FAM-66, which displayed double recombinants in all seven children during female meiosis, was excluded from the analysis. In general, apparent double recombination events within short intervals of the genome most likely are the consequence of genotyping problems, although in very rare cases balanced translocations arisen during oogenesis could also cause such haplotype patterns.

To improve linkage analysis methods for complex diseases caused by pseudoautosomal genes, reliable genetic maps in these regions are crucial. Genetic maps increasing in length have been observed in telomeric and subtelomeric regions of different human chromosomes, reflecting a higher recombination rate per physical length unit in these regions, reviewed by Rouyer et al. [24]. PAR1 exhibits these subtelomeric features in a particular way. Genetic maps in males are markedly longer than genetic maps in females, as inferred here from the comparison of male and female recombination events and from multipoint linkage data. The biological process for these differences is not yet well understood, but they exist and should be considered in genetic studies using sex-specific maps instead of sex-averaged maps. The human $\mathrm{X}$ chromosome exhibits some peculiarities compared to the autosomes chromosomes since females have two X chromosomes and males only one. Based on our map, it is determined how genetically different, in genetic size, the female $\mathrm{X}$ chromosome is from the male $\mathrm{X}$ chromosome. The genetic map of the male X chromosome can be considered only where crossovers occur. The genetic length is therefore very short $(56 \mathrm{cM})$ because the $\mathrm{X}$ chromosome can only recombine with the $\mathrm{Y}$ chromosome in the PARs. The genetic map of the $\mathrm{X}$ chromosome in females is much longer because the two $\mathrm{X}$ chromosomes can recombine on their entire length, the genetic length is estimated to be about $198 \mathrm{cM}$. For this reason, the human sex chromosomes cannot be treated like the autosomes when trying to map sex linked genes.

Comparisons between our map and those already published for PAR1 revealed that our map provides statistically different estimates for genetic distances. The genetic maps proposed by Rouyer and by Lien were the only ones that did not display significant differences when comparing the whole PAR1. Nevertheless the latter showed a significant shorter genetic length close to the telomere and close to the PAB1. These differences could be due to the fact that close to the Xp telomere the Lien's estimates are based on only 3 genetic markers while our estimates are based on 11 genetic markers. The same conclusion holds close to the PAB1, this last interval is only covered with 2 genetic markers in Lien's map and 7 markers were used for our estimates. The differences found in the maps suggested by Rutgers and by Schmitt could be due to the lack of genetic markers at the first $750 \mathrm{~kb}$ failing to account for recombination events in this regions and resulting then in a much shorter genetic length. Therefore, we conclude that the new sex-specific map presented in this work is based on the largest set of genetic markers in the PARs and thus represents the most accurate resource for obtaining genetic map information for these two regions.

To date, one of the most accurate and widely used genetic maps for the human genome is the Rutgers' map v2. However, this map shows a weakness in the PARs since a low number of genetic markers was considered in these two regions. To the improvement of genetic mapping projects we will integrate our new sex-specific map for the PARs in the Rutgers' map v.2. Although there is still a long way to go for understanding the exact mechanisms and the precise function(s) of the PARs, the new genetic map presented here could be a first step to mapping new pseudoautosomal genes responsible for complex diseases. It is hoped that researches will take these two regions into proper account when performing genome-wide studies. So far, the PARs are effective gaps in genome-wide analyses. In our view, in the context of such efforts, screening for pseudoautosomal linkage should not be neglected.

\section{Acknowledgement}

A.F was supported by a grant of the Nationales Genomforschungsnetz NGFN-01GR0465. We thank Dr. Matise from the Department of Genetics, Rutgers University, for allowing us to use their genotype data from the pseudoautosomal regions.

\section{Electronic-Database Information}

CEPH database: http://cephb.fr

Ensembl Genome browse: http://www.ensembl.org

National Center for Biotechnology Information (NCBI): http://www.ncbi.nlm.nih.gov

dbSNP database: http://www.ncbi.nlm.nih.gov/SNP

International Hapmap project: http://www.hapmap.org 


\section{References}

1 Rouyer F, Simmler MC, Johnsson C, Vergnaud G, Cooke HJ, Weissenbach J: A gradient of sex linkage in the pseudoautosomal region of the human sex chromosomes. $\mathrm{Na}$ ture 1986;319:291-295.

$\checkmark 2$ Freije D, Helms C, Watson MS, Donis-Keller $\mathrm{H}$ : Identification of a second pseudoautosomal region near the $\mathrm{Xq}$ and $\mathrm{Yq}$ telomeres. Science 1992;258:1784-1787.

-3 Flaquer A, Rappold GA, Wienker TF, Fischer C: The human pseudoautosomal regions: a review for genetic epidemiologists. Eur J Hum Genet 2008;16:771-779.

$\checkmark 4$ Ross MT, Grafham DV, Coffey AJ, et al: The DNA sequence of the human $\mathrm{X}$ chromosome. Nature 2006;434:325-337.

5 Blaschke RJ, Rappold G: The pseudoautosomal regions, SHOX and disease. Curr Opin Genet Dev 2006;16:233-239.

6 Dietter J, Mattheisen M, Fürst R, Rüschendorf F, Wienker TF, Strauch K: Linkage analysis using sex-specific recombination fractions with GENEHUNTER-MODSCORE. Bioinformatics 2007;23:64-70.

7 Maniatis N, Collins A, Xu CF, McCarthy LC, Hewett DR, Tapper W, Ennis S, Ke X, Morton NE: The first linkage disequilibrium (LD) maps: Delineation of hot and cold blocks by diplotype analysis. Proc Natl Acad Sci USA 2002;99:2228-2233.

>8 Tapper W, Collins A, Gibson J, Maniatis N, Ennis S, Morton NE: A map of the human genome in linkage disequilibrium units. Proc Natl Acad Sci USA 2005;102:1183511839.
9 Ziegler A, König IR: A Statistical Approach to Genetic Epidemiology: Concepts and Application, ed 1. Weinheim, Wiley-VCH, 2006.

10 Li N, Stephens M: Modeling linkage disequilibrium and identifying recombination hot spots using single-nucleotide polymorphism data. Genetics 2004;165:2213-2233.

11 Arnheim N, Li HH, Cui XF: PCR analysis of DNA sequences in single cells: single sperm gene mapping and genetic disease diagnosis. Genomics 1990;8:415-419.

12 Henke A, Fischer C, Rappold GA: Genetic map of the human pseudoautosomal region reveals a high rate of recombination in female meiosis at the $\mathrm{Xp}$ telomere. Genomics 1993; 18:478-485.

13 Vergnaud G: No increase in female recombination frequency in the distal part of the pseudoautosomal region. Genomics 1994; 24:610-612.

14 Rouyer F, Simmler M-C, Vergnaud G, Johnsson C, Levilliers J, Petit C, Weissenbach J: The pseudoautosomal region of the human sex chromosomes. Cold Spring Harb Symp Quant Biol 1986;51:221-228.

15 Lien S, Szyda J, Schechinger B, Rappold G, Arnheim N: Evidence for heterogeneity in recombination in the human pseudoautosomal region: high resolution analysis by sperm typing and radiation-hybrid mapping. Am J Hum Genet 2000;66:557-566.

16 Schmitt K, Vollrath D, Foote S, Fisher EM, Page DC, Arnheim N: Four PCR-based polymorphisms in the pseudoautosomal region of the human $\mathrm{X}$ and $\mathrm{Y}$ chromosomes. Hum Mol Genet 1993;2:1978.
17 Matise TC, Chen F, Chen W, De La Vega FM, Hansen M, He C, Hyland FC, Kennedy GC, Kong X, Murray SS, Ziegle JS, Stewart WC, Buyske S: A second-generation combined linkage physical map of the human genome. Genome Res 2007;A:1783-1786.

18 International HapMap-Consortium: A haplotype map of the human genome. Nature 2005;437:1299-1320.

19 Daw EW, Thompson EA, Wijsman EM: Bias in multipoint linkage analysis arising from map misspecification. Genet Epidemiol 2000; 19:366-380.

20 Fingerlin TE, Abecasis GR, Boehnke M: Using sex-averaged genetic maps in multipoint linkage analysis when identity-by-descent status is incompletely known. Genet Epidemiol 2006;30:384-396.

21 O'Connell JR, Weeks DE: Pedcheck: A program for identification of genotype incompatibilities in linkage analysis. Am J Hum Genet 1998;63:259-266

22 Rappold GA, Klink A, Weiss B, Fischer C: Double crossover in the human Xp/Yp pseudoautosomal region and its bearing on interference. Hum Mol Genet 1994;3:1337-1340.

23 Lander ES, Green P: Construction of multilocus genetic linkage maps in humans. Proc Natl Acad Sci USA 1987;84:2363-2367.

24 Rouyer F, de la Chapelle A, Andersson M, Weissenbach J: An interspersed repeated sequence specific for human subtelomeric regions. EMBO J 1990;9:505-514. 